\title{
Green Management Model for Eco-farm in Thailand
}

\author{
Suthet Chandrucka $^{1, *}$, Nuttiya Tantranont ${ }^{1}$, Manat Suwan ${ }^{2}$ \\ ${ }^{1}$ Asian Development College for Community Economy and Technology, Thailand \\ ${ }^{2}$ Graduate School, Chiang Mai Rajabhat University, Thailand
}

Copyright (C) 2015 by authors, all rights reserved. Authors agree that this article remains permanently open access under the terms of the Creative Commons Attribution License 4.0 International License

\begin{abstract}
The purposes of this research were to study the potential of a local community in producing organic crops and to develop patterns of green management. The instruments employed for data collection included field surveys, in-depth interviews, questionnaires, observations, focus group discussions and a community seminar at Mae-Rim District, Chiang Mai Province, Thailand. The results were that the community had the potential to produce organic plants, as it situates mostly on a flatland by the mountain, with fertile soil abundantly filled with minerals and nutrients. In terms of social dimension, people in the community had three sets of knowledge, namely, organic plantation management, economic community, and the new Theory of Sufficiency Economy. Integrating with their economic and marketing network, they consequently formulated the SALUANG Model which illustrates knowledge and experience sharing of all stakeholders in the agricultural management, transparency of the management that allows everyone to be aware of the original sources and all the process that meet quality and safety standards, integration of local wisdom with modern knowledge that emphasizes in environmentally friendly toxin-free agriculture and participatory resources conservation, understanding of customer needs, accessibility to the knowledge body by focusing at information exchange among members and with outsiders, establishment of a collaborative network comprising farmers who favor green agriculture, good governance, and well-being of the community.
\end{abstract}

Keywords Green Standard, Green Management, Agricultural Supply

\section{Introduction}

In the past 10 years, the agricultural sector of Thailand has excessively relied on the use of chemicals, and the trend of chemical import kept increasing every year. The quantity of herbicides used by Thai farmers is at a very high level. This can lead to high risks, and other detrimental effect on the health and financial return to the farmers. Currently, the average cost of herbicide use is accounted for about $10 \%$ of the total rice production cost. For other crops, the cost can be even higher. For example, chemical use for strawberry production can cost as much as $32 \%$ of the total cost. [1] Therefore, herbicides pose a major public health concern in Thailand. According to a survey by the Occupational and Environmental Disease Division, Disease Control Department, Ministry of Public Health, Thai farmers were found to be prone to contact with herbicides for as high as $53.76 \%$. In addition, from studying the in-patient data of Ministry of Public Health, the substances that are responsible for most illness cases were organophosphates and herbicides. [2] In addition, these chemicals also affect wellness of the ecosystem by, for example, spreading into the air and contaminating surface water and groundwater. Although local people have had experience in growing toxin-free vegetables for their household consumption and for selling within the community, their green agriculture at the commercial level lacked appropriate management and cooperation. Without proper planning on crop growing in each season, it would be difficult for their products to find the market and, thus, only earn low selling prices. Therefore, this research's objective is to develop a green management model for agricultural production via cooperation of farmers in Mae Rim District, Chiang Mai Province, in order to develop a new concept of fair administration that meets international standards.

\section{Materials and Methods}

This qualitative research was conducted by means of explorations, interviews, and focus group meetings. The collected data were then summarized with descriptive explanations in order to explore and develop a green management agricultural model that is appropriate with the area of Saluang Sub-district, Mae Rim District, Chiang Mai. For this development, the five-step approach in knowledge management of Panich [3] was applied as a basic concept for managing the knowledge body in this research. The five 
steps consist of 1) defining the knowledge, by making questions that will lead to the understanding of causes of the problem, requiring aspects of knowledge, and sources of knowledge required to obtain a solution; 2) creating knowledge via opinion sharing, exploring evidence-based reasoning, and collaboratively finding an approach to solve the problem; 3) extracting and capturing knowledge, by structuring the acquired knowledge and organizing it as a database for systematic storage with appropriate categories; 4) knowledge sharing, which is a key component of knowledge management by preparing and providing documents in case of explicit knowledge and organizing seminars or discussion floors in case of tacit knowledge; and 5) using the obtained knowledge by applying it to make decisions on solving the problem and to enhance knowledge and experience regarding green agricultural management. These steps should be repeated in cycles in order to enhance the knowledge endlessly.

Field surveys were conducted in order to study the situation and collect data through the community leaders, representatives of farmers, and agricultural officers of Mae Rim District, Chiang Mai. The informants were purposively sampled by selecting persons who can profoundly explain and provide information about their agriculture experiences. This research process was divided into three main steps.

Step 1 was studying the context of knowledge and collecting useful knowledge, which divided into 2 phases. Phase 1 was studying the explicit knowledge which was about the theories, research papers or information available within the community and elsewhere that have been collected as the directions to create the knowledge management model to manage suitable agricultural production for the areas in the next step. Phase 2 was studying the tacit knowledge by the in-depth interviews with 3 main groups included 1) The group of 15 farmers included agricultural sages and community leaders to gain information about the local wisdom for agricultural production, the style of yield management, marketing and ability to be the nontoxic and organic agricultural producers. 2) The group of 10 food processing included entrepreneurs and products collectors to gain information about the production standard, cooking standard, distribution, delivery, raw material procurement standard, demand for agricultural products, price, and knowledge and understanding about food hygiene and nutrition. 3) The group of 20 consumers included students, teachers and staff within Chiang Mai Rajabhat University to gain information about the production standard, distribution, delivery, decision to purchase, understanding, and awareness of the dangers of toxic residues in the food. Meanwhile, there was also the study of non-participatory research from farmers in the area, and the incidents were recorded together with summarizing the content to conclude the traditional knowledge. After that, such traditional knowledge was discussed in terms of agriculture, the use of renewable energy in the manufacturing processes, transportation, and worthiness of the product cost, health affected on producers and consumers, management problems, marketing, and sustainable solutions for community by the community. There were 12 discussion participants included farmer representatives, agricultural sages, and qualified experts or personnel from various sectors to provide knowledge and supports.

Step 2 was creating the knowledge management model about the toxin-free agricultural production or green management by applying the local participation and sharing knowledge to all parties to create the green management model from the combination of traditional knowledge, new knowledge and information technology, as well as the concept of related theories, such as organic agriculture, Good Agricultural Practices (GAP) standard, Good Manufacturing Practices (GMP) standard, green supply chain management, traceability system, and the sufficiency economy philosophy, which is the philosophy of the east that focused on sufficiency, life-sufficient understanding, and faithful livelihood causing the farmers to live happily and not indulge with the consumerism by the current globalization. This new knowledge was integrated comprehensively with the local wisdom that had been synthesized to derive the green standards and the model that were appropriate to the community and complied with the lifestyle of the local people.

Step 3 concerned with the model verification. The model developed via collaboration of all parties was verified by nine selected persons. They included one academic expert, one agricultural guru, four farmer representatives, one agriculturist, and one entrepreneur representative. These selected persons attended a seminar organized for them to criticize the green management model regarding its efficiency when considering capacity and abilities of the community in managing their green products. The knowledge and specified standards in the model were verified to make sure that the green management model was appropriate for the community and allowed the people to solve their agricultural problems in a sustainable manner.

The collected data were also examined for their accuracy. These included data and knowledge related to the process of toxin-free agricultural production. The synthesized data were verified by using a technique called triangulation. With this technique, different sources of data were used. The verification was done by the key informants, together with the researcher, interpreting the data systematically from their traces to ensure that the derived facts were accurate and reliable.

\section{Conclusions}

\subsection{Results}

\subsubsection{According to the Study}

The general characteristics of the farmers living in 
Saluang, Khi Lek and Huay Sai of Mae Rim district in Chiangmai, most of them gain agricultural knowledge from their experiences and traditional knowledge which passed down from their ancestors. Most of the revenues are between $60,001-80,000$ Baht per year, depending on the production area and productivity each year. Also, there are the middlemen who will come to purchase the crops at the farmland, so most of the farmers do not sell their crops directly by themselves.

In term of the availability of the area, the data collections from the local government representative and field research found that there are total 281,085.62 hectares of the area of Mae Rim District, while 78,120.75 hectares are agricultural areas, about 26,954.53 hectares are rice paddies, and the rest is crop farms. Most residents work in agriculture. The most dominant fruits and vegetables are rice, sticky rice, sweet pepper, carrot, chayote, kale, cabbage, tomato, cucumber and corn. The areas are foothill plains which have plenty of minerals and water. The climate condition is ideal for the growth of the plants. Also, there are various supports for the toxin-free agriculture during the past few years. However, the farmers who need to sell their crops will be affected by the problems about the price and marketing, so some of them will still need to do the chemical agricultures.

In terms of the potential of the toxin-free agricultures, the data collection from the Agriculture Office of Mae Rim in Chiangmai in December, 2012 found that the local farmers were able and ready to do the non-toxin primary food farming. In 2012, the farmers from three parishes of the Mae Rim district could produce 28.9 tons of organic vegetables per year out of the whole vegetables quantity which was 675.5 tons per the year. Also, there was 5,280 tons of the organic rice agriculture per year, which could produce up to 5,200 tons of organic rice per year.

In terms of the social background, it were found that some of the agricultural community begins to make the toxin-free agriculture because the health problems which are the impacts of the chemical agriculture. Therefore, they must adjust the production by themselves with their existing knowledge which inherited from their ancestors through the long experience of farming, and the experience of organic planting for household consumption, which providing them the potential to make the toxin-free agriculture for distribution to the needs of the markets.

In terms of potential of the community, the highlights of the community can be classified into 7 aspects as following: 1) The community leaders have acceptable visions, are supported by the communities, and have strong bargaining power to coordinate with all parties. 2) In term of society, community member is bound to help each other as relatives, the senior systems play a significant role, and religious activities come to associate. 3) In term of location, the convenient transportation, there are routes to various districts and provinces in the north, also have a suitable environment for cultivation. 4) In term of participation, community members participate in expressing their opinions and making decisions. 5) In term of economy, community run the economic integration includes the main job (farming, gardening) and part time job by applying local knowledge to make money, for example, handcrafts, hand woven fabrics, basketry, home stays, and establishing the saving groups and village funds. 6) In term of the coordination, there are several communication channels within the community, including individual communicating through the meeting representatives, voice broadcast tower in the village, information board, and community radio. 7) In term of local tradition, there is the conservation of the inherited local traditions and customs, passing on the cultures from a generation to the next generation, and applying the culture and tradition as the medium for the cooperation in various activities.

In terms of the knowledge management, it were found that there are 4 aspects of community knowledge as following: 1) Knowledge about the management of organic cultivation, conservation of soil, water and forest, plant selection for planting in the next season, using organic and natural ways to increase the nutrients to the soil and water and reduce the pests, agroforestry systems management, and crop rotation are the examples. 2) Knowledge about the local traditions and beliefs which are the transfer process of knowledge that latent in the forms of various cultures, Kwankgaw tradition, paying homage to the goddess of grain, culture of community involvement, and integration activities are the examples. 3) Knowledge about the local economy, which are community demand and supply, family accounting and resource management. 4) The community has the understanding of the agricultural principles on the basis of the king's sufficiency economy philosophy and community through actual practices, repetitive manufacturing, and exchanging the official and unofficial knowledge. However, there is a lack of continuous integration and no effective information storage database system.

The comparison between the demand and the amount of agricultural products from the interview with 10 all kinds of food entrepreneurs in the cafeteria of Rajabhat University in Chiangmai which is the main target market of this model found that they consumed 100.2 tons per year by average demand for vegetables compared to the total weight of agricultural products. Meanwhile, the community can produce up to 675.5 tons per year, which means the community can also distribute those exceeded products to other markets, such as hospital, hotel, shopping mall, etc.

\subsubsection{Creating and Developing the Green Management Model}

From the development of the toxin-free agricultural production of the community, it was found that a style that suits the area can be summarized as the "SALUANG MODEL" which consists of 7 key elements as following: S (share) refers to all parties participate in the exchanging and sharing of ideas, experiences and resources with each other, and then apply them in their areas appropriately. A 
(awareness) refers to creating the awareness of the dangers of using chemicals that can affect the overall health of the community, and having the strong sense of maintaining product quality and safety standard which is the creation of the social responsibility. L (local wisdom) refers to the transfer and integration of the local traditional knowledge which has its identity and values as the combination of the traditional knowledge and the new knowledge together to develop the existing knowledge, to improve the levels of learning, and to effectively apply such knowledge. U (understanding customer need) refers to the access and understanding of the demands of customers or consumers. A (assess) refers to providing the opportunities for accessing to the knowledge, and focusing on exchange and dissemination of information and knowledge between community members and outsiders, which will help in creating the understanding and relationship networks with other organizations. $\mathrm{N}$ (natural environment friendly and social responsibility) refers to focusing on the green agriculture, which is environmentally friendly and socially responsible. G (governance) refers to management by the involvement of all parties with faithfulness, transparency and fairness.

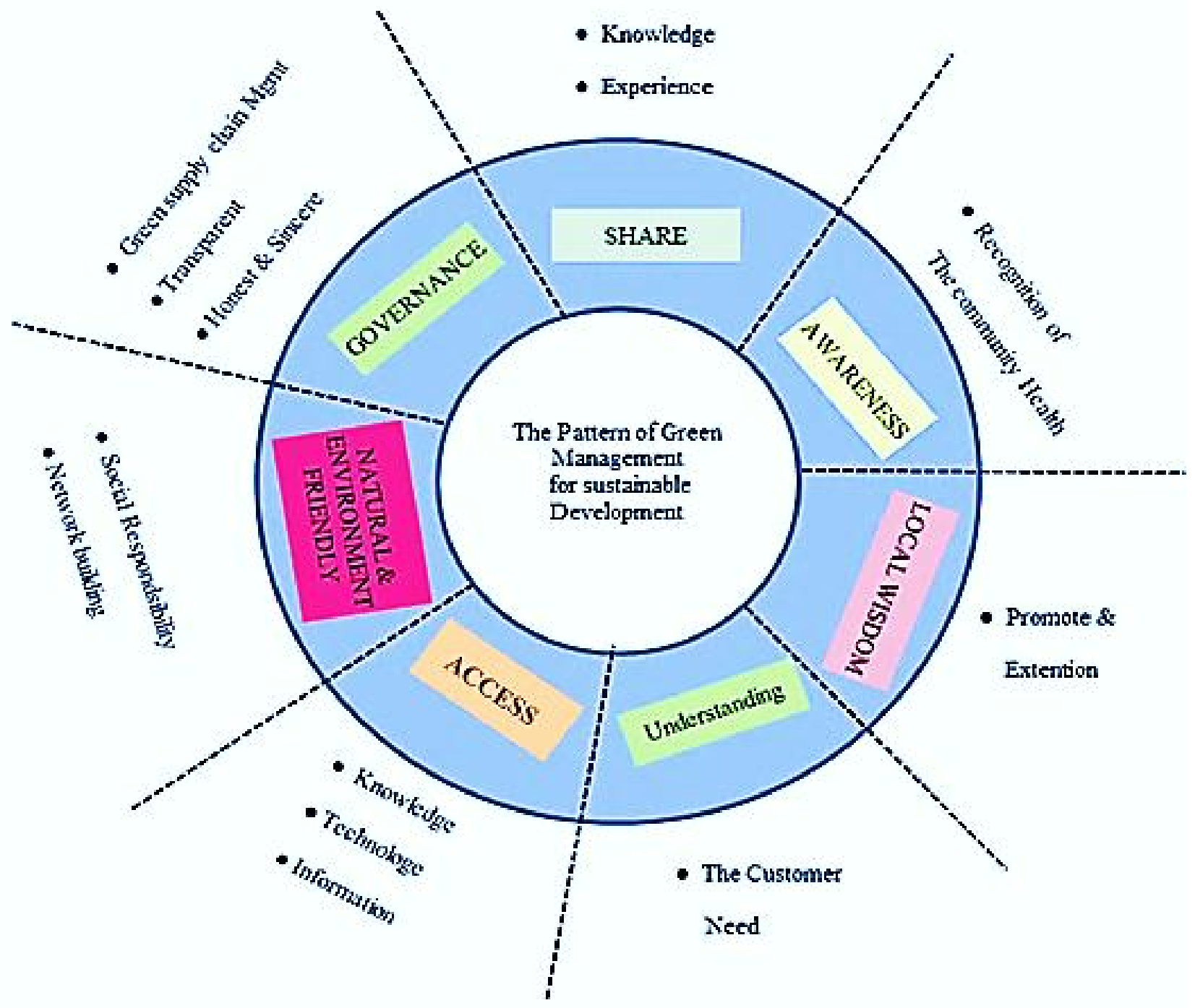

SALUANG MODEL

Figure 1. The green management model appropriate with the community and its sustainable development called the "SALUANG Model" 
By the way, there is a part of the 7 key elements which is the transfer and integration of the traditional knowledge which has its identity and values passed on to the next generation, which is a part of the L (local wisdom) that only appeared in the studied community. Meanwhile, the lesson learned from the community forum found that, in term of farmers and involved people, Share is the most important, which is calculated as $82 \%$. The meaning is that everyone participates in management together to develop their own community by expressing ideas, exchanging knowledge, experiences and resources with each other. Next, the Governance is calculated as $64 \%$ or considered as the second importance because it is the element to build up the trust of consumers and those involved, which driven by 3 elements include the Green Supply chain Management as the integrated supply chain management process which is the characteristic of linking related business together with disclosed, transparent and honest communication, the information about productivity source and shipping from the origin or productivity source to the consumers can be checked through the product tag or product Barcode. The rest elements are Natural \& Network building 55\%, Understanding $45 \%$, Awareness $41 \%$, Local wisdom $36 \%$ and Access $27 \%$ respectively.

"SALUANG MODEL" The Pattern of Green Management for sustainable Development has developed with many similarities with the definition based on the concept of the International Federation of Organic Agriculture Movement-IFOAM which summarized the meaning of the green agriculture that it is "The production system that provides priority to the sustainable of soil health, ecology and people by relying on the ecological processes, biodiversity and the cyclical nature of the special characteristics of each different areas. Instead of using factors of production with negative effects, the green and organic agriculture combines local knowledge, innovations and scientific knowledge to conserve the environment, supports the fair relationships, and improves the quality of life of everyone and creatures involved". Also, it is compliance with the integrated supply chain model based on the concept of Bowersax, Closs and Cooper [5] that said the integrated supply chain management is an appearance of the business alliance network into the cooperative strategy management model which has its longtime goal by every sectors pay attention to the cooperative process to improve the efficiency and reduces the cost. Furthermore, there is also a part that associated with the model of "Green business Models in Nordic Region" [6] who has given the definition of the green business model, which is the business model that supports the development of products and services that can provide environmental benefits, reduce energy consumption and waste which have less environmental impact than the traditional business model.

\section{Green Management Model}

From the 7 elements of SALUANG MODEL, there are further studies for the suitable model to be applied in the green management. The summarized results are that the business management model under the green supply chain can be divided into 3 steps of the main activities.

1. The upstream activity is the activity of production level of the supply chain causing from the partnership between local farmer network by having the Enterprise Development Parish Center and community learning center as the link, coordinating for information exchange, plan participation, and operating the import logistics, as well as the farm certification by the Department of Agricultural Extension.

2. The midstream activity is the activity of business alliance under the agreement on the plan, logistics collecting, privatization, packing, and distribution within the network.

3. The downstream activity is the activity of product distribution network or export logistic between the toxin-free agriculture network and the business alliances to distribute the products through market channels to the target customers, and to be a source of information for further business management.

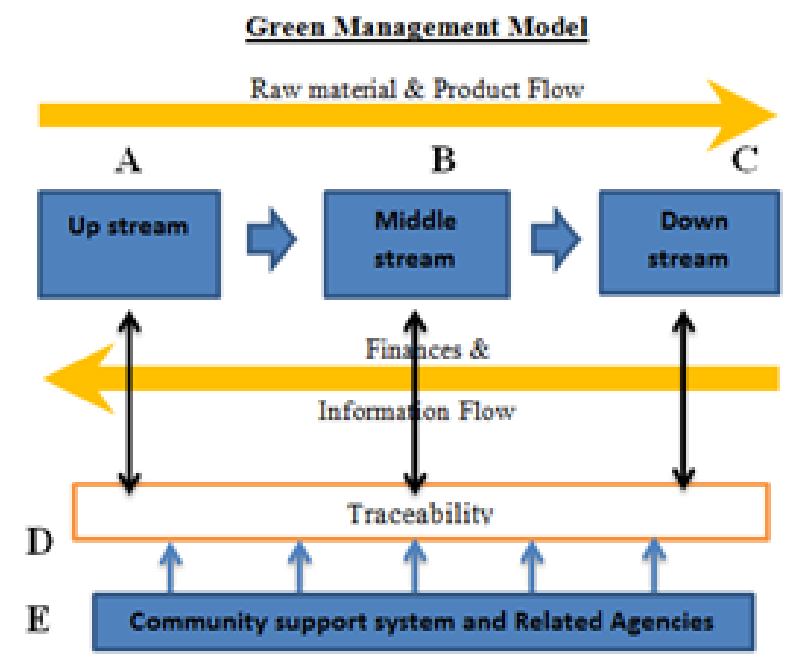

Figure 2. Green Management Model and relevant components of an agricultural production management system

Also, there is the Green Supply Chain Management (GSCM) which is suitable for creating a collaborative network to manage and improves performances from the raw material management, information technology management from supplier, to the customers by considering the environmental impact that can incur during various processes, and focus on working together closely throughout the product lifecycle to increase the efficiency in all processes [7]. By the way, GSCM consists of 2 elements include traceability system and logistics system, which help improving the safety and quality to meet the standard specifications, which in accordance with the modern management concept called Value Chain [8]. 
TRACKIN0: The isspection of raw materials to the last consarmer for prodact recalls.

IR.ACNO: Traceability frcen the last consumer to rav materials, to determine the cause of an arer in the product.

Figure 3. Traceability throughout the entire production process

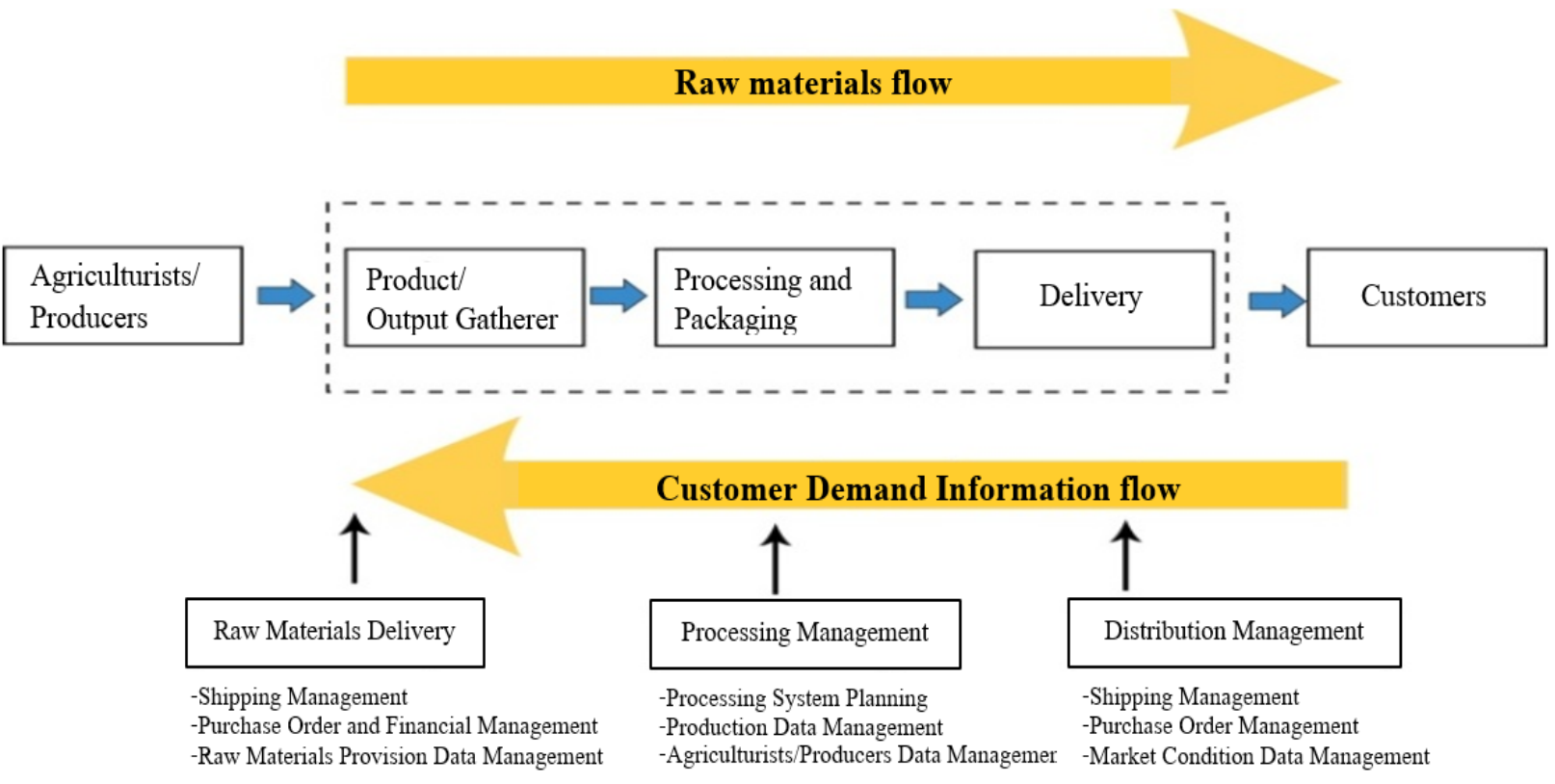

Figure 4. Elements of Logistic System and Agricultural Outputs Management 


\section{Business Model for Non-Toxic Agricultural Supply Chain}

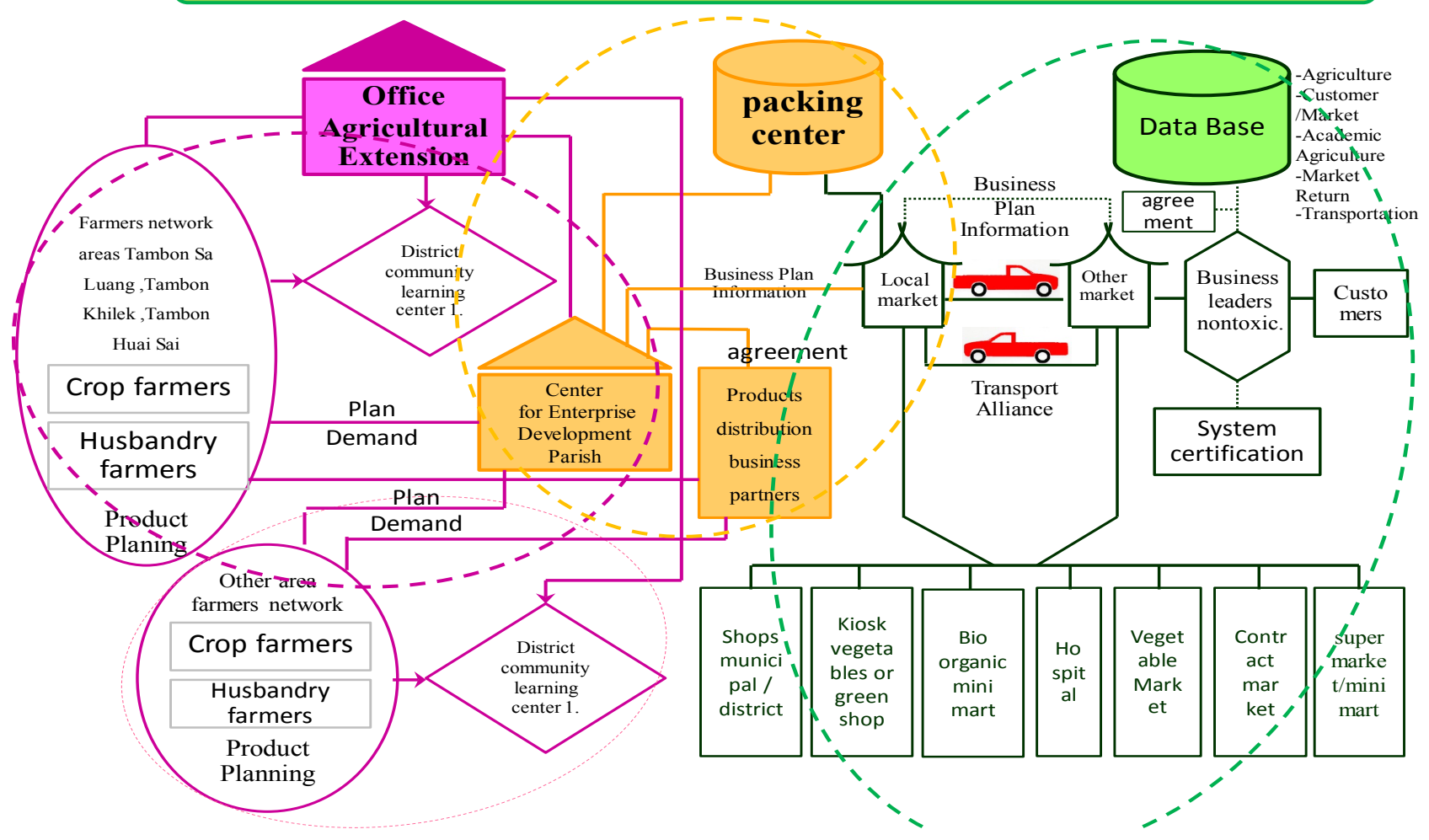

Figure 5. The business management model under the supply chain of green products

In summary, there are 4 processes of the business model under the green agricultural supply chain which will point out the synergies between the business alliances in the implementation of activities under the supply chain (Figure 5) as following: 1) Demand process is in a production level of the supply network, which is the area preparation, high quality raw materials and seed selection, as well as utilities standard, such as water resources and so on. 2) Integrating the supply chain is transferring information to members to have the cultivating plan to meet the demand of the market by determining who will grow each kind of plant, duration of planting and harvesting in accordance with the amount and production potential of each different person, as well as planning the packaging, transportation, bar code and distribution among the farmers network. 3) Marketing is the distribution of product through various marketing channels to the target market based on the agreed upon business plans by having supporting system, including the evaluation of operations, human resource management, community network, and customer satisfaction. And 4) Social outcomes is to develop and support local wisdom by applying the new technology together with the traditional knowledge appropriately causing the farmer's community to have steady income, better life quality, and strengthen the community.

\subsection{Discussion}

In order to apply the 7 elements of "SALUANG MODEL" to solve problems and manage the holistic green production appropriately in the modern community where there is the rapid changes, people in the community need to be aware of and understand the overview of common problems, including the "SALUANG MODEL" which should be applied effectively. By the important factor that will contribute the successful green production management of "SALANG MODEL" is the participation and coordination of all involved people, also the supports from public agencies and academic institutions are required. The study found that there is no plan to solve the green agricultural production problems in the past because there is no one who started to determine the solutions. Therefore, it is important to create a shared vision of people in the area by the community leaders to change and collaborate with both public sector and private sector to achieve the common consciousness until it becomes tradition, then, the vision is ready to be performed in actual situations to create the common learning process, share knowledge with each other, develop knowledge, and apply the knowledge to the green agricultural production beneficially, or even apply the local wisdom to increase revenue and create economic opportunities for community, such as hand woven fabrics group, eco-tourism, home stays with farming activities. The idea above is consistent with the concept of [3] who said that creating the vision about knowledge and creating organizational knowledge management team by beginning with the existing intellectual capital, and seeking for more knowledge will cause the learning atmosphere, the well-being of community members 
from their participation in the community management, and preserve the natural resources which will lead to the better life quality and improve the community economy.

There is also the use of available social capital to increase the values, for example continue to conserve the tradition and culture is the local attractions for people from other areas to visit, and apply the culture as the medium to create the collaboration within the community. The idea is consistent with the concept of United Nation (1975) [10] that said participation is the process about practice which associated with various levels of public, for example the process of decision making which is about the social purpose, and resource management, also the participation is the voluntary action to various activities and projects in the community which allow people to participate in the initiative, decision making, collaboration, and sharing responsibility for several matters that affects the people themselves. In addition, social outcome is the application of wisdom, good quality of life and the strength of the community.

The results of this study are different from [13], Their studies found that there are 2 models of the supply chain which are the traditional supply chain and the supply chain for export manufacturing. The majority of farmers have troubles in production, marketing and need the supports from involved agencies for the price guarantee. From this study, the suggestions are to encourage the farmer's integration to deal the product under the contract for quality of production which is different from SALUANG MODEL that keeps the community members together to think of the way to manage the production by themselves from their own experiences to solve the manufacturing, marketing and pricing problems. Lastly, the voluntarily participation is required and ready to expand to other communities in order to strengthen the career without any pressure from the outside but the decision of the community members.

Such development process is the summary of the concrete knowledge management of Saluang, KhiLek and HuaySai of Mae Rim district in Chiangmai, which is the main idea, management theory, knowledge that can be adjusted to guide the knowledge management for others community as well. Therefore, the farmers that can produce the toxin-free product to exceed the demand of Mae Rim Rajabhat University, and want to distribute their products to other markets, they will needed to apply the "SALUANG MODEL" to manage the operation, as well as the product must maintain the quality and safety standard, all processes can be checked, production and transportation is considered to have less impact on the environment and high social responsibility, pricing and decision making are done by the participation of all parties, and work with integrity, transparency and fairness to everyone to be the successful and sustainable solutions.

\subsection{Conclusions}

In conclusion, the area potential survey found that the area is suitable for various kind of plant cultivation, and the farmers also have knowledge about the organic agriculture as well. However, there is a lack of knowledge storage system, and a lack of continuous supports which result in unsteady works. So, some farmers use chemical fertilizers, chemical pesticides and synthetic plant hormones in agriculture causing several problems endlessly, such as soil degradation, lack of fertility, outbreaks of disease and insect problems, loss of biodiversity in agricultural plots, toxic residues in the environment, and the problem of expensive fertilizer and chemical which causing high investment, while the output price does not rise proportionately to meet the higher costs, thus the farmers lose their profits and increase their liabilities, as well as increase the risk of health problems of both farmers and consumers due to the toxic residues in agricultural product and environment.

The solution studies found that some farmers have turned to do toxin-free agriculture or green and organic farming which is another way that can solve the problems effectively because it is the way to avoid the uses of synthetic substances, but focusing on the natural material, such as using natural manure and compost, green manure, and bio fertilizer to nourish the fertile soil, encourage the crop rotation, create diverse ecological balance which is considered as the effective benefits for natural resources, and also is a way to reduce the risk of diseases and insects pest outbreaks, as well as lets the natural enemies control crop pests without using any chemicals causing a balance in agricultural ecosystems in another way. In term of food safety, the development of new agricultural theory to support integrated agriculture based on the demand of community to reduce chemical usage and increase the values of agricultural product, which accordance with the King's sufficiency economy [12] which is the practical guide to live a sufficient life with rational, prudence and careful life, have revenue, reduce expenses, and save the money to use when needed.

Combing with the latest knowledge in the green agricultural production supply chain management, we can begin to bring the market demand to plan the common process of each sector, The good examples are determining the time to harvest, product collecting, packaging, shipping and distribution to customers or markets. Each step is considered about the impact on health, environment and society. There is the honest, transparent and fair operation which can be checked through every process by the traceability system which is the management system by all involved parties that invented by the community members as the green agricultural production model to suits the area called "SALUANG MODEL".

This integrated green agricultural supply chain is the further development of local wisdom and modern technology that will lead the society to the targets which are the social stability and strong economy which will improve the life for people in the community to the society and the country at last. The presentation of such business model is aimed at the long-time goals by linking them into 5 dimensions, including 
economic dimension, social dimension, ethical dimension, health dimension, and environmental dimension which consistent with the green agricultural production management model that is suitable for community and is the model for other communities to further develop and apply to suits their local conditions of each different areas which truly is the sustainable development of the communities.

\section{Acknowledgements}

We would like to express our sincere thanks towards volunteer researchers who devoted their time and knowledge in the implementation of this project.

Nevertheless, we express our gratitude toward our families and colleagues for their kind co-operation and encouragement which help us in completion of this project.

\section{REFERENCES}

[1] A. Khusanthia. Situation of agricultural chemicals, Risk and impact overall, Surveillance of agricultural chemicals Conference, Program support the food security, Jul, 2007.

[2] P. Itsaraphan, Situations of illness with the use of pesticides in Thailand, Surveillance of agricultural chemicals Symposium, Jun, 2011.

[3] W. Panich. Knowledge management: wisdom created under health assurance for all, Knowledge Management Institute,
Bangkok, 2004.

[4] P. Suriya, P. Dummai. Interview, Mae Rim District, Chiang Mai, Mar 31, 2012.

[5] D. Bowersox, D. Closs, M. Cooper. Supply Chain Logistic Management. $2^{\text {nd }}$ Edition, Mcgraw Hill International, New York, 2007.

[6] Danish Enterprise and Construction Authority. Green business models in the Nordic region, Online available from http://www.motiva.fi/files/4878/Greenpaper_Green_Busines s_Models_in_the_Nordic_Region_A_Key_to_Promote_Sust ainable_Growth.pdf.

[7] P. Seriphong. Greening Supply Chain Management, The National Productivity Institute, Bangkok, 2009.

[8] S. Pothong. Traceability, Online available from http://www.logisticsdigest.com/education/article/item/5966

[9] W. Wothirakun. Systems development, supply chain management, food safety, Department of Industrial Promotion, Ministry of Industry, Bangkok, 2007

[10] Y. Wutthimethim. Principles of community development and community development. Anukhrothai, Bangkok, 1983.

[11] M. Tracey, (1998). The Importance of Logistics Efficiency to Customer Service and Firm Performance, The International Journal of Logistic Management, Vol.9, No 2.

[12] S. Tantiwetkun. Sufficiency Economy, Journal Rajabhat the Old City, Vol.14, No 2, 1-11.

[13] A. Sungsri-in, K. Lertrat, S. Khawchaimaha. Fresh Vegetable Supply Chain in Nakorn Pathom Province, Khon Kaen University, Khon Kaen, 2009. 